\title{
Quelques enjeux des villes-frontières Introduction au cahier thématique
}

\section{Bernard Reitel, Mulhouse, Olivier Graefe, Bayreuth}

En tant que limite de souveraineté, la frontière est associée à un territoire continu et clairement délimité, édifié par un Etat moderne (ANDERSON 1996). L'ordre mondial érigé progressivement depuis le traité de Westphalie (1648) est celui d'un monde borné, composé de territoires stato-nationaux, qui entretiennent des relations internationales et où chaque ville appartient avant tout à un système national de villes (Moreau Defarges 1998). Une ville divisée par une frontière, comme Berlin ou Jérusalem, représente un cas atypique. Les villes en position frontalière sont des cas plus fréquents, mais la frontière est avant tout perçue comme une barrière, «un objet géographique qui met de la distance dans la proximité» (ARBARETSchulz et al. 2004) et imprègne durablement l'espace urbain.

Or, cette vision semble à présent dépassée en raison des transformations qu'ont connues les limites des Etats depuis plusieurs décennies. L'émergence d'une société de l'information qui privilégie une extension continue des réseaux de circulation et de communication ainsi que le processus de mondialisation qui y est associé se traduisent par des recompositions spatiales à de multiples échelles. Les villes et les frontières constituent des révélateurs de ces changements globaux (Reitel et al. 2002). Dans un contexte de mobilité, de croissance des flux de marchandises, d'informations et de capitaux, de nombreuses frontières ont acquis une grande porosité (Newman 2003): elles semblent avoir évolué du stade de barrière à celui de contact (RatTi \& Reichman 1993). Elles sont devenues des lignes de contact mettant non seulement en relation des territoires limitrophes, mais aussi de plus en plus le lointain (Piermay 2005). Dans le même temps, les espaces urbains concernés par les frontières se caractérisent par l'existence de processus de fragmentation. Les ségrégations sont basées sur la résidence (BASSAND 1997), mais aussi sur le mouvement et traduisent l'émergence de frontières d'un nouveau type: le rapport au temps introduit ainsi de nouvelles disparités entre les zones connectées où la vitesse et l'urgence sont la norme et les espaces interstitiels laissés à l'écart où le temps est ralenti (Piermay \& Zander 2007). L'existence de nombreuses interactions économiques et sociales favorise l'émergence de «milieux transfrontaliers" caractérisés par des interdépendances et des complémentarités (Martinez 1994). Les agglomérations transfrontalières, ces espaces urbains traversés par une ou plusieurs frontières, apparaissent comme les symboles de ces mouvements, non seulement sur la frontière entre le Mexique et les EtatsUnis, sur les frontières des Etats européens, mais également et de manière croissante dans d'autres régions du monde.

La frontière présente une dimension symbolique et culturelle: elle renvoie à des représentations, à des constructions identitaires qui permettent de dissocier le «nous» des «autres» (Donnan \& WILSON 2005: PAASI 1996) et qui donnent corps au territoire. Ce contexte de développement des interactions transfrontalières se traduit-il par des dynamiques d'intégration ou, au contraire, assiste-t-on à une volonté d'affirmation et de renforcement des identités territoriales? La frontière transgressée ou dépassée produit-elle de l'invention, de la stimulation, débouche-t-elle sur des territorialités nouvelles? Comment les dynamiques globales dans lesquelles s'inscrivent les Etats s'articulent-elles avec les stratégies des acteurs urbains qu'ils soient politiques, sociaux ou économiques?

En tant que zones de concentrations d'acteurs variés aux stratégies complexes, les espaces urbains constituent des lieux de maximisation des interactions sociales à l'échelle du Monde (LÉvy 1998). De ce fait, les villes en situation de frontière font l'objet d'un intérêt croissant. Les quatre articles empiriques présentés dans ce numéro thématique apportent quelques éléments d'information aux questions posées. Ils montrent que si les Etats fixent les règles générales, il existe toutefois un jeu que les acteurs locaux peuvent mettre à leur profit en raison de leur bonne connaissance de l'environnement local. Des figures hybrides sont édifiées sur la frontière évoquant selon le cas la traduction (BAlibar 2003) ou le paradoxe (BAREL 1984).

L'article de Bernard Reitel propose une méthode pour analyser le degré d'intégration de 15 agglomérations transfrontalières situées sur les frontières du territoire français dans un contexte général de construction européenne. Cette analyse montre qu'un processus de territorialisation est en cours notamment dans les plus grandes agglomérations, celles qui disposent des ressources humaines, politiques et financières pour élaborer une stratégie de dépassement de la frontière. Cette dernière est alors considérée avant tout comme un instrument d'articulation. Les collectivités coopérant à l'échelle de ces agglomérations peuvent être 
assimilées à des territoires d'entre-deux. La démarche doit beaucoup aux incitations de l'Union Européenne (UE). Favorisée par la proximité géographique entre les acteurs publics locaux, elle reste cependant fragile en raison de la prégnance des systèmes nationaux.

Freerk J. Boedeltje, Henk van Houtum et Olivier T. KRAMSCH présentent leurs raisonnements concernant l'ouverture de la frontière entre les deux parties de l'île de Chypre séparées depuis 1974, sur la ville divisée de Nicosie. L'intégration de la partie méridionale à l'Union Européenne s'est traduite à partir de 2003 par une ouverture progressive de la ligne de séparation restée hermétique pendant près de 30 ans. Les deux parties de l'île ont donc connu un développement différent et s'ignoraient mutuellement. Les habitants de chaque composante territoriale ont profité de cette ouverture pour saisir des opportunités et les flux transfrontaliers ont connu une augmentation rapide, notamment entre les deux parties de la ville divisée. Le développement des interactions renforce le sentiment de différenciation des populations au détriment du rapprochement prôné par l'UE, d'autant que la frontière conserve une forte dimension symbolique, mettant l'Occident face au Moyen-Orient. Nicosie apparaît comme une entité paradoxale où se combinent confrontation et complémentarité.

ThOMAS LundÉN interroge quant à lui l'impact des contextes géopolitiques sur les comportements des populations à l'échelle locale. Il propose une comparaison entre trois agglomérations transfrontalières de petite taille, aux caractéristiques différentes sur le plan matériel, fonctionnel et historique. Dans tous les cas, la frontière apparaît comme un objet de protection et de différenciation symbolique et identitaire, mais aussi politique, y compris à Haparanda-Tornio, entre Suède et Finlande, où l'intégration est forte et ancienne et où la coopération entre les collectivités locales est élaborée.

Olivier Walther analyse les relations économiques entre trois villes situées sur la frontière du Niger, du Bénin et du Nigeria. Les acteurs majeurs de ces villes sont les marchands qui ont contribué à construire les centralités des villes de Gaya, Malanville et Kamba: ils jouent à la fois sur les grands réseaux d'échanges, principalement méridiens, qui animent l'espace sahélien et sur les différences des politiques publiques menées par les Etats pour développer les marchés. L'espace transfrontalier est caractérisé par l'existence de complémentarités et de concurrences qui fluctuent au gré des politiques publiques. La frontière est un instrument de régulation exploité par les marchands dans un contexte incertain, celui des situations économiques et politiques de chacun des Etats qui disposent d'une faible maitrise de leurs territoires.
IzHAK SCHNell propose un essai portant sur la place des frontières et des villes dans un Monde en pleine mutation. Il observe que le Monde est organisé par les centralités et les flux qui semble consacrer une perte du monopole de l'Etat sur son propre territoire. En partant de l'idée que les frontières constituent à la fois des objets d'inclusion et d'exclusion, et qu'un système d'acteurs et d'institutions multiples est à l'œuvre, il considère l'émergence de frontières à différents niveaux géographiques. Ces frontières osmotiques constituent de véritables paradoxes et activent chacune leur propre filtrage, qu'il présente à travers plusieurs exemples qui dénotent la capacité de mobilisation dont font preuve les acteurs urbains: la frontière apparaît ambivalente, tantôt risque et contrainte, tantôt opportunité et ressource.

Plus que jamais, les mutations de notre Monde semblent associer frontière et ville. Dans les espaces urbains transfrontaliers, la limite de souveraineté, même transgressée et traversée par de nombreux flux, conserve une forte signification pour les acteurs. Quant aux grandes agglomérations urbaines, elles sont devenues des espaces où les propriétés attribuées classiquement aux frontières, telles que le filtrage, la différence ou l'affirmation symbolique, sont de plus en plus présentes.

\section{Bibliographie}

Anderson, M. (1996): Frontiers: territory and state formation in the modern world. - Cambridge: Cambridge University Press.

Arbaret-Schulz, C., Beyer, A., Piermay, J.-L., Reitel, B., Selimanovski, C., Sohn, C. \& P. Zander (2004): La frontière, un objet en mutation. - In: Espacestemps.net, http://espacestemps.net/document842.html 15.12.2005. BaLIBAR, E. (2003): Europe as Borderland. - http://www. ru.nl/socgeo/colloquium/Europe \%20as\%20Borderland. pdf 1.6.2005.

BAREL, Y. (1984): Le paradoxe et le système. Essai sur le fantastique social. - Grenoble: Presses Universitaires.

BASSAND, M. (1997): Métropolisation et inégalités sociales. - Lausanne: Presses Polytechniques et Universitaires Romandes.

Donnan, H. \& T. Wilson (eds) (2005): Culture and power at the edges of the state. National support and subversion in European border regions. - Münster: LiT Verlag.

LÉvy, J. (1998): Le tournant géographique. Penser l'espace pour lire le monde. - Paris: Belin.

Martinez, O. (1994): The dynamics of border interaction. New approaches to border analysis. - In: SCHOFIELD, C. (ed.): Global boundaries. World boundaries. - Londres: Routledge: 4-16.

Moreau Defarges, P. (1998): L'ordre mondial. - Paris: Armand Colin. 
Newman, D. (2003): On borders and power. A theoretical framework. - In: Journal of borderlands studies 18, 1:13-25.

PAASI, A. (1996): Territories, boundaries and consciousness. The changing geographers at the finnish-russian border. - Chichester: John Wiley.

Piermay, J.-L. \& P. Zander (2007): Espace urbain et temporalités. - In: KAHN, R. (dir.): Régulation temporelle et territoires. - Paris: L'Harmattan (sous presse). Piermay, J.-L. (2005): La frontière et ses ressources: regards croisés. - In: Antheauame, B. \& F. Giraut (éds): Le territoire est mort. Vive les territoires! Une (re)fabrication au nom du développement. - Paris: IRD: 203-222.

Ratti, R. \& S. Reichman (1993): Theory and practice of transborder cooperation. - Basel: Helbing \& Lichtenhahn.

Reitel, B., Zander, P., Piermay, J.-L. \& J.-P. Renard (éds) (2002): Villes et frontières. - Paris: EconomicaAnthropos.

Dr. Bernard Reitel, Centre de recherche sur les économies, les sociétés, les arts et les techniques (CRESAT), Université de Haute-Alsace, 10, rue des Frères Lumière, F-68093 Mulhouse Cedex, France.

e-mail: bernard.reitel@uha.fr

Dr. Olivier Graefe, Lehrstuhl für Bevölkerungs- und Sozialgeographie, Universität Bayreuth, Universitätsstrasse 30, D-95447 Bayreuth.

e-mail: olivier.graefe@uni-bayreuth.de 